\title{
HOMOMORPHISMS OF COMMUTATIVE CANCELLATIVE SEMIGROUPS INTO NONNEGATIVE REAL NUMBERS
}

BY

\author{
MOHAN S. PUTCHA AND TAKAYUKI TAMURA
}

\begin{abstract}
Let $S$ be a commutative cancellative semigroup and $T_{0}$ be a cofinal subsemigroup of $S$. Let $h_{0}$ be a homomorphism of $T_{0}$ into the semigroup of nonnegative real numbers under addition. We prove that Kobayashi's condition [2] is necessary and sufficient for $h_{0}$ to be extended to $S$. Further, we find a necessary and sufficient condition in order that the extension be unique. Related to this, the "boundedness condition" is introduced. For further study, several examples are given.
\end{abstract}

1. Introduction. A commutative cancellative archimedean idempotent-free semigroup is called an $\Re$-semigroup. Kobayashi [2] proved the following:

ThEOREM 1.1. Let $T_{0}$ be a subsemigroup of an $\Re$-semigroup $S$ and let $h_{0}$ be a homomorphism of $T_{0}$ into the semigroup $\mathbf{R}_{+}^{0}$ of nonnegative real numbers under addition. Then $h_{0}$ can be extended to a homomorphism of $S$ into $\mathbf{R}_{+}^{0}$ if and only if the pair $\left\langle T_{0}, h_{0}\right\rangle$ satisfies the following condition: if $x, y \in T_{0}$ and $x \mid y$ ( $x$ divides $y$ ) in $S$, then $h_{0}(x) \leqslant h_{0}(y)$.

One of the authors [4] has studied the homomorphisms of $T_{0}$ into $\mathbf{R}_{+}$from the viewpoint of positive quasi-orders. In this paper, we treat the homomorphisms of $T_{0}$ into the nonnegative real numbers in the case when $S$ is a commutative cancellative semigroup and $T$ is its subsemigroup. Theorem 2.1 will be a straightforward generalization of the classical result that characters can be extended from a subgroup of an abelian group $G$ to $G$ itself. In $\S 2$, we will show that Theorem 1.1 holds if $T_{0}$ is cofinal in $S$. In $\S 3$, we will introduce a "boundedness condition" and discuss the relation between this condition and the extension of a homomorphism beyond a filter. In $\$ 4$, we will give a few examples, which show that Theorem 2.1 does not necessarily hold if $T_{0}$ is not cofinal.

A subsemigroup $U$ of a commutative semigroup $S$ is called unitary in $S$ if

Received by the editors November 13, 1974.

AMS (MOS) subject classifications (1970). Primary $20 \mathrm{M} 99$.

Key words and phrases. Commutative cancellative (idempotent-free) semigroup, cofinal subsemigroup, unitary subsemigroup, homomorphism, $K$-condition, $B$-condition (boundedness condition), unitary closure, filter, filter closure. 
$x \in S, a \in U$ and $a x \in U$ imply $x \in U$. $U$ is called cofinal in $S$ if, for every $x$ $\in S$, there is a $y \in S$ such that $x y \in U$. As is well known, see [1] or [5], a unitary cofinal subsemigroup $U$ induces a group congruence $\rho_{U}$ on $S$ defined by $x \rho_{U} y$ if and only if $a x=b y$ for some $a, b \in U$. We denote $S / \rho_{U}$ by $S / U$. Furthermore the kernel of $S \rightarrow S / U$ coincides with $U$.

Let $T$ be a nonempty subsemigroup of $S$. The smallest unitary subsemigroup $\bar{T}$ of $S$ containing the subsemigroup $T$ is called the unitary closure of $T$ in $S . \bar{T}$ is given by

$$
\bar{T}=\{x \in S: x t \in T \text { for some } t \in T\} \text {. }
$$

A nonempty subsemigroup $F$ of $S$ is called a filter of $S$ [3] if $x, y \in S$ and $x y$ $\in F$ implies $x, y \in F$. The smallest filter $\widetilde{T}$ of $S$ containing the subsemigroup $T$ is called the filter closure of $T$ in $S$. Then

$$
\widetilde{T}=\{x \in S: x y \in T \text { for some } y \in S\} .
$$

(1.2) The following hold.

(1.2.1) $T \rightarrow \bar{T}$ and $T \rightarrow \widetilde{T}$ are closure mappings, that is, $T \subseteq \bar{T}, T \subseteq \widetilde{T}$. $T_{1} \subseteq T_{2}$ implies $\bar{T}_{1} \subseteq \bar{T}_{2}$ and $\widetilde{T}_{1} \subseteq \widetilde{T}_{2}$.

$\overline{\bar{T}}=\bar{T}, \widetilde{T}=\widetilde{T}$.

(1.2.2) $\bar{T}$ is unitary in $S, \widetilde{T}$ is a filter in $S$, and $T$ is cofinal in $\widetilde{T}$.

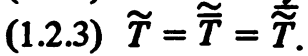

(1.2.4) $\bar{T} \subseteq \widetilde{T}$ and $\bar{T}$ is unitary cofinal in $\widetilde{T}$.

Throughout this paper, $\mathbf{R}$ denotes the set of real numbers, $R$ the set of rational numbers, $\mathbf{R}_{+}\left(\mathbf{R}_{-}\right)$the set of positive (negative) real numbers; $\mathbf{R}_{+}^{\mathbf{0}}$ $\left(R_{-}^{0}\right)$ the set of nonnegative (nonpositive) real numbers; $Z_{+}\left(Z_{-}\right)$the set of positive (negative) integers and $Z_{+}^{0}\left(Z_{-}^{0}\right)$ the set of nonnegative (nonpositive) integers.

If $S$ is a semigroup and if $X$ is a subsemigroup of the additive group $R$, then the notation $\operatorname{Hom}(S, X)$ denotes the semigroup of homomorphisms of $S$ into $X$ under the usual operation. Let $X_{1}, X_{2}, Y_{1}$ and $Y_{2}$ be commutative semigroups such that $X_{1} \subseteq X_{2}$ and $Y_{1} \subseteq Y_{2}$. Let $h_{1} \in \operatorname{Hom}\left(X_{1}, Y_{1}\right)$ and $h_{2} \in \operatorname{Hom}\left(X_{2}, Y_{2}\right)$. If $h_{2} \mid X_{1}=h_{1}$, we say that $h_{1}$ of $\operatorname{Hom}\left(X_{1}, Y_{1}\right)$ is extended to $h_{2}$ of $\operatorname{Hom}\left(X_{2}, Y_{2}\right)$; in particular, if $Y_{1}=Y_{2}$, we say that $h_{1}$ of $\operatorname{Hom}\left(X_{1}, Y_{1}\right)$ is extended to $X_{2}$. If the extension $h_{2}$ of $h_{1}$ of $\operatorname{Hom}\left(X_{1}, Y_{1}\right)$ to $X_{2}$ is unique, we say that $h_{1}$ of $\operatorname{Hom}\left(X_{1}, Y_{1}\right)$ is uniquely extended to $X_{2}$. Let $h \in \operatorname{Hom}(S, R)$. $h$ is called trivial if $h(x)=0$ for all $x \in S$.

In this paper the binary operation in a commutative semigroup will be denoted by addition, i.e. + . 
2. Extensions from cofinal subsemigroups. In this section, we will prove the following generalization of Theorem 1.1.

THEOREM 2.1. Let $T_{0}$ be a cofinal subsemigroup of a commutative cancellative semigroup $S$ and let $h_{0}$ be a homomorphism of $T_{0}$ into the additive semigroup $\mathbf{R}_{+}^{0}$ of nonnegative real numbers. Then $h_{0}$ can be extended to $S$ if and only if

$$
t_{1} \in S+t_{2} \text { implies } h_{0}\left(t_{1}\right) \geqslant h_{0}\left(t_{2}\right) \text { for all } t_{1}, t_{2} \in T_{0} \text {. }
$$

In this paper, the condition $(K)$ will be called the $K$-condition. It is obvious that if $h_{0}$ can be extended to $S$ then the $K$-condition must hold. We will prove sufficiency. Let $X$ denote the set of pairs $\langle T, h\rangle$ where $T$ is a subsemigroup of $S$ containing $T_{0}$ and $h \in \operatorname{Hom}\left(T, \mathrm{R}_{+}^{0}\right)$ such that $h \mid T_{0}=h_{0}$ and $\langle T, h\rangle$ satisfies the $K$-condition.

Let $[a]$ be the cyclic subsemigroup generated by $a$ and let $[T, a]$ be the subsemigroup generated by $T$ and $a$, i.e.,

$$
[T, a]=T \cup(T+[a]) \cup[a] .
$$

LEMMA 2.2. Let $\langle T, h\rangle \in X$ and suppose that $a \in S$ and $(T+[a]) \cap T$ $\neq \varnothing$. Then there exists $h^{\prime}:[T, a] \rightarrow \mathbf{R}_{+}^{0}$ such that $\left\langle[T, a], h^{\prime}\right\rangle \in X$. Further, $h^{\prime}$ is unique.

Proof. There exist $t_{1}, t_{2} \in T, N \in Z_{+}$such that $t_{1}=N \cdot a+t_{2}$. Then $h\left(t_{1}\right) \geqslant h\left(t_{2}\right)$ by the K-condition. Define $h^{\prime}:[T, a] \rightarrow \mathbf{R}_{+}^{0}$ by

$$
\begin{aligned}
h^{\prime}(t+n \cdot a) & =h(t)+\frac{n}{N}\left[h\left(t_{1}\right)-h\left(t_{2}\right)\right], & & t \in T, n \in Z_{+}^{0} \\
h^{\prime}(n a) & =\frac{n}{N}\left[h\left(t_{1}\right)-h\left(t_{2}\right)\right], & & n \in Z_{+} .
\end{aligned}
$$

First we show that $h^{\prime}$ is well defined: $t+n \cdot a=t^{\prime}+n^{\prime} \cdot a, t, t^{\prime} \in T, n, n^{\prime} \in$ $Z_{+}$, implies $N \cdot t+N n \cdot a+\left(n+n^{\prime}\right) \cdot t_{2}=N \cdot t^{\prime}+N n^{\prime} \cdot a+\left(n+n^{\prime}\right) \cdot t_{2}$, that is, $N \cdot t+n \cdot t_{1}+n^{\prime} \cdot t_{2}=N \cdot t^{\prime}+n^{\prime} \cdot t_{1}+n \cdot t_{2}$. This shows $h^{\prime}(t+n \cdot a)$ $=h^{\prime}\left(t^{\prime}+n^{\prime} \cdot a\right)$, hence $h^{\prime}$ is well defined. From its definition, $h^{\prime}$ is clearly a homomorphism into $\mathrm{R}_{+}^{0}$, and $h^{\prime} \mid T=h$. Assume $t+n \cdot a=s+t^{\prime}+n^{\prime} \cdot a$ for some $a \in S$. Then $N \cdot t+n \cdot t_{1}+n^{\prime} \cdot t_{2}=N \cdot s+N \cdot t^{\prime}+n^{\prime} \cdot t_{1}+$ $\boldsymbol{n} \cdot \boldsymbol{t}_{\mathbf{2}}$ which implies

$$
N \cdot h(t)+n \cdot h\left(t_{1}\right)+n^{\prime} \cdot h\left(t_{2}\right) \geqslant N \cdot h\left(t^{\prime}\right)+n^{\prime} \cdot h\left(t_{1}\right)+n \cdot h\left(t_{2}\right)
$$

by the $K$-condition. This gives $h^{\prime}(t+n \cdot a) \geqslant h^{\prime}\left(t^{\prime}+n^{\prime} \cdot a\right)$. Hence $\left.\llbracket T, a\right]$, $\left.h^{\prime}\right\rangle \in X$. If $h^{\prime \prime}$ is any extension of $h$ to a homomorphism of $[T, a]$ into $R_{+}^{0}$, we must have 


$$
h\left(t_{1}\right)=h^{\prime \prime}\left(t_{1}\right)=N \cdot h^{\prime \prime}(a)+h^{\prime \prime}\left(t_{2}\right)=N \cdot h^{\prime \prime}(a)+h\left(t_{2}\right)
$$

so that $h^{\prime \prime}(a)=N^{-1}\left[h\left(t_{1}\right)-h\left(t_{2}\right)\right]=h^{\prime}(a)$. It follows that $h^{\prime \prime}(t+n \cdot a)=$ $h^{\prime}(t+n \cdot a)$ for all $t \in T$, all $n \in Z_{+}$, that is, $h^{\prime \prime}=h^{\prime}$. .

To consider the case when $(T+[a]) \cap T=\varnothing$, we need a lemma. From now on, $t_{1}, t_{2}, t_{3}$ and $t_{4}$ will denote arbitrary elements of $T$.

Let

$$
\begin{aligned}
& A(a)=\left\{n^{-1}\left[h\left(t_{2}\right)-h\left(t_{1}\right)\right]: t_{1}+n \cdot a \in t_{2}+S\right\}, \\
& B(a)=\left\{n^{-1}\left[h\left(t_{2}\right)-h\left(t_{1}\right)\right]: t_{2} \in t_{1}+n \cdot a+S\right\} \text {, }
\end{aligned}
$$

where $a \in S, A(a)$ and $B(a)$ mean the sets depending on $a$. Note that $0 \in A(a)$ and hence $A(a) \neq \varnothing$.

LEMMA 2.3. Let $\langle T, h\rangle \in X$ and suppose that $(S+[a]) \cap T \neq \varnothing$. Then $\operatorname{Sup} A(a) \leqslant \operatorname{Inf} B(a)<\infty$.

Proof. Since $(S+[a]) \cap T \neq \varnothing$, there are $t_{1}, t_{2} \in T, x \in S$ and $n \in Z_{+}$ such that $t_{2}=t_{1}+n \cdot a+x$. Hence $B(a) \neq \varnothing$ and $\operatorname{Inf} B(a)<\infty$. Suppose $t_{1}+$ $n_{1} \cdot a=t_{2}+s_{1}$ and $t_{4}=t_{3}+n_{2} \cdot a+s_{2}$ where $n_{1}, n_{2} \in Z_{+}, s_{1}, s_{2} \in S$. Then $n_{1} \cdot t_{4}+n_{2} \cdot t_{1}=n_{1} \cdot t_{3}+n_{2} \cdot t_{2}+n_{2} \cdot s_{1}+n_{1} \cdot s_{2}$. By the K-condition, $n_{1} \cdot h\left(t_{4}\right)+n_{2} \cdot h\left(t_{1}\right) \geqslant n_{1} \cdot h\left(t_{3}\right)+n_{2} \cdot h\left(t_{2}\right)$. Hence $n_{2}^{-1}\left[h\left(t_{4}\right)-h\left(t_{3}\right)\right] \geqslant$ $n_{1}^{-1}\left[h\left(t_{2}\right)-h\left(t_{1}\right)\right]$. Thus we get $\operatorname{Sup} A(a) \leqslant \operatorname{Inf} B(a)$.

LemmA 2.4. Let $\langle T, h\rangle \in X$ and suppose that $(S+[a]) \cap T \neq \varnothing$ but $(T+[a]) \cap T=\varnothing$. Then $h$ can be extended to a homomorphism $h^{\prime}:[T, a]$ $\rightarrow \mathbf{R}_{+}^{0}$ and $\left\langle[T, a], h^{\prime}\right\rangle \in X$. The $h^{\prime}$ is determined by choosing $h^{\prime}(a)$ such that $\operatorname{Sup} A(a) \leqslant h^{\prime}(a) \leqslant \operatorname{Inf} B(a)$. Moreover, every extension $h^{\prime \prime}$ of $h$ to $[T, a]$ such that $\left\langle[T, a], h^{\prime \prime}\right\rangle \in \mathrm{X}$ is obtained in this way.

Proof. Choose $b \in \mathbf{R}_{+}^{0}$ such that

$$
\operatorname{Sup} A(a) \leqslant b \leqslant \operatorname{Inf} B(a) .
$$

Define

$$
\begin{aligned}
h^{\prime}(t+n \cdot a) & =h(t)+n \cdot b & & \text { for } t \in T, n \in Z_{+}^{0} . \\
h^{\prime}(n a) & =n b & & \text { for } n \in Z_{+} .
\end{aligned}
$$

Since $S$ is cancellative and $(T+[a]) \cap T=\varnothing$, every element of $T+[a]$ is uniquely expressed as $t+n \cdot a$ and hence $h^{\prime}$ is well defined. Then $h^{\prime}$ is clearly a homomorphism $[T, a] \rightarrow \mathrm{R}_{+}^{0}$ and $h^{\prime} \mid T=h$. Suppose that $t_{1}+n_{1} \cdot a=t_{2}+$ $n_{2} \cdot a+s, n_{1}, n_{2} \in Z_{+}^{0}, s \in S$. Then there are three possibilities: $n_{1}=n_{2}, n_{1}$ $>n_{2}$ and $n_{1}<n_{2}$. If $n_{1}=n_{2}$, then, since $S$ is cancellative, $t_{1}=t_{2}+s$, hence $h\left(t_{1}\right) \geqslant h\left(t_{2}\right)$ by the K-condition. This implies $h^{\prime}\left(t_{1}+n_{1} \cdot a\right) \geqslant h^{\prime}\left(t_{2}+n_{2} \cdot a\right)$. 
If $n_{1}>n_{2}$, then $t_{1}+\left(n_{1}-n_{2}\right) \cdot a=t_{2}+s$ and, by the choice of $b$,

$$
h\left(t_{2}\right)-h\left(t_{1}\right) \leqslant\left(n_{1}-n_{2}\right) \cdot b \text {. }
$$

This implies

$$
h^{\prime}\left(t_{2}+n_{2} \cdot a\right)=h\left(t_{2}\right)+n_{2} \cdot b \leqslant h\left(t_{1}\right)+n_{1} \cdot b=h^{\prime}\left(t_{1}+n_{1} \cdot a\right) .
$$

If $n_{1}<n_{2}$, then $t_{1}=t_{2}+\left(n_{2}-n_{1}\right) \cdot a+s$. By the choice of $b$,

$$
\left(n_{2}-n_{1}\right) \cdot b \leqslant h\left(t_{1}\right)-h\left(t_{2}\right)
$$

This gives

$$
h^{\prime}\left(t_{2}+n_{2} \cdot a\right)=h\left(t_{2}\right)+n_{2} \cdot b \leqslant h\left(t_{1}\right)+n_{1} \cdot b=h^{\prime}\left(t_{1}+n_{1} \cdot a\right) .
$$

Therefore $\left\langle[T, a], h^{\prime}\right\rangle \in X$.

Assume that $h^{\prime \prime}$ is an extension of $h$ to $[T, a]$ and that $t_{1}+n_{1} \cdot a=t_{2}+$ $s_{1}$ and $t_{4}=t_{3}+n_{2} \cdot a+s_{2}, n_{1}, n_{2} \in Z_{+}, s_{1}, s_{2} \in S$. Using the assumption that $h^{\prime \prime}$ obeys the K-condition, $t_{1}+n_{1} a=t_{2}+s$ gives $h^{\prime \prime}\left(t_{1}\right)+n_{1} h^{\prime \prime}(a) \geqslant$ $h^{\prime \prime}\left(t_{2}\right)$, so that $h^{\prime \prime}(a) \geqslant\left(h\left(t_{2}\right)-h\left(t_{1}\right)\right) / n_{1}$, hence $h^{\prime \prime}(a) \geqslant \operatorname{Sup} A(a)$. Likewise we have $h^{\prime \prime}(a) \leqslant\left(h\left(t_{4}\right)-h\left(t_{3}\right)\right) / n_{2}$, hence $h^{\prime \prime}(a) \leqslant \operatorname{Inf} B(a)$. By the former half of the lemma, $\left\langle[T, a], h^{\prime \prime}\right\rangle \in X . \square$

COROLlARY 2.5. In Lemma 2.4 , the extension $h^{\prime}$ is unique if and only if

$$
\operatorname{Sup} A(a)=\operatorname{Inf} B(a)
$$

Proof of Sufficiency of Theorem 2.1. Define the partial order in $X$ by $\left\langle T_{1}, h_{1}\right\rangle \leq\left\langle T_{2}, h_{2}\right\rangle$ if and only if $T_{1} \subseteq T_{2}$ and $h_{2}$ is an extension of $h_{1}$ to $T_{2}$. Then it is easy to see that $X$ satisfies the condition for Zorn's lemma and so $X$ has maximal members. To show that any such maximal member has domain $S$, it suffices to show that if $\langle T, h\rangle \in X$ and $a \notin T$, then $h$ can be extended to $h^{\prime}:[T, a] \rightarrow \mathbf{R}_{+}^{0}$ such that $\left\langle[T, a], h^{\prime}\right\rangle \in X$. Since $T$ is cofinal, $(S+[a]) \cap$ $T \neq \varnothing$, furthermore there are two possibilities: $(T+[a]) \cap T \neq \varnothing$ and $(T+$ [a]) $\cap T=\varnothing$. Lemma 2.2 has dealt with the first case; Lemma 2.4 has done the second case. Thus the theorem has been proved. $\square$

COROLlary 2.6. Let $S$ be a commutative cancellative semigroup and $T_{0}$ a unitary cofinal subsemigroup of $S$. Then every homomorphism $h$ of $T_{0}$ into $\mathbf{R}_{+}^{0}$ can be extended to $S$.

Proof. Every $h$ satisfies the $K$-condition.

Corollary 2.7. Let $T_{0}$ be an ideal of $S$. Then every homomorphism $h$ of $T_{0}$ into $\mathbf{R}_{+}^{0}$ can be uniquely extended to $S$. 
Proof. Lemma 2.2 is applied to this case since $\left(T_{0}+[a]\right) \cap T_{0} \neq \varnothing$ for each $a \in S$. The direct alternate proof of this corollary is left for the reader's exercise. $\square$

Since every subsemigroup of a commutative archimedean semigroup is cofinal, Theorem 1.1 is a special case of Theorem 2.1.

THEOREM 2.8. Let $T$ be a cofinal subsemigroup of a commutative cancellative subsemigroup $S$, and let $h: T \rightarrow \mathbf{R}_{+}^{0}$ be a homomorphism. Then $h$ admits $a$ unique extension to $S$ if and only if, for each $a \in S, \operatorname{Sup} A(a)=$ $\operatorname{Inf} B(a)$.

Proof. Assume $h$ admits a unique extention to $S$. Then $\langle T, h\rangle$ satisfies the $K$-condition. Suppose that $h_{1}$ and $h_{2}$ are distinct extensions such that $\left\langle[T, a], h_{1}\right\rangle$ and $\left\langle[T, a], h_{2}\right\rangle$ obey the K-condition for some $a \notin T$. Then $(S+$ [a]) $\cap T \neq \varnothing$ since $T$ is cofinal in $S ;(T+[a]) \cap T=\varnothing$ by Lemma 2.2. Now Lemma 2.4 shows that $\left\langle[T, a], h_{1}\right\rangle$ and $\left\langle[T, a], h_{2}\right\rangle$ are in $X$. By Theorem 2.1, $h_{1}$ and $h_{2}$ can be extended to homomorphisms $h_{1}^{\prime}: S \rightarrow R_{+}^{0}$ and $h_{2}^{\prime}: S \rightarrow \mathbf{R}_{+}^{0}$ respectively; but $h_{1}^{\prime} \neq h_{2}^{\prime}$. This contradicts the assumption. Therefore $h$ admits a unique extension to $[T, a]$ for each $a \notin T$. If $(T+[a]) \cap T \neq \varnothing$, we can easily show that if $n \in Z_{+}, t_{1}, t_{2} \in T$ and $t_{1}+n \cdot a=t_{2}$, then

$$
\operatorname{Sup} A(a)=\operatorname{Inf} B(a)=\left(h\left(t_{2}\right)-h\left(t_{1}\right)\right) / n \text {. }
$$

If $(T+[a]) \cap T=\varnothing$, then Corollary 2.5 shows $\operatorname{Sup} A(a)=\operatorname{Inf} B(a)$.

Conversely, suppose $\operatorname{Sup} A(a)=\operatorname{Inf} B(a)$ for every $a \in S$. If $t_{2}=t_{1}+s$, $t_{1}, t_{2} \in T, s \in S$, then $2 t_{2} \in 2 t_{1}+s+S$, which implies

$$
\operatorname{Inf} B(s) \leqslant 2\left[h\left(t_{2}\right)-h\left(t_{1}\right)\right] \text {. }
$$

As $t_{1}+2 \cdot s \in t_{2}+S, \operatorname{Sup} A(s) \geqslant 1 / 2\left[h\left(t_{2}\right)-h\left(t_{1}\right)\right]$. Hence

$$
1 / 2\left[h\left(t_{2}\right)-h\left(t_{1}\right)\right] \leqslant 2\left[h\left(t_{2}\right)-h\left(t_{1}\right)\right] \text {. }
$$

It follows that $h\left(t_{2}\right) \geqslant h\left(t_{1}\right)$. Hence $h$ satisfies the $K$-condition, and so $h$ is extended to $S$. By Lemma 2.2 and Corollary 2.5 , the extension is unique since $\operatorname{Sup} A(a)=\operatorname{Inf} B(a)$ for each $a \in S$.

3. Boundedness condition. In Lemma 2.3, we see that the set $A$ is bounded. In light of this, we will introduce the boundedness condition (B-condition). In this section, we assume that $S$ is a commutative cancellative semigroup and let $P=S F$ where $P$ is a prime ideal, $P \neq \varnothing$, and $F$ is a filter [3], $F \neq \varnothing$. Let $a \in$ $P$. The subsemigroup of $S$ generated by $a$ and $F$ is denoted by $P_{F}(a)$ or $\mathrm{P}(a)$ if $F$ is fixed. We define the relation $\rho$ on $P$ as follows: $c \rho d$ if and only if $m \cdot c$ $+s=n \cdot d+t$ for some $s, t \in F$ and some $m, n \in Z_{+}$. Then $\rho$ is an equiva- 
lence relation on $P$ and each $\rho$-class is a subsemigroup of $P$; i.e., $\rho$ has the following properties:

(3.1.1) $x \rho y$ implies $x \rho x+y$ for all $x, y \in P$.

(3.1.2) $x \rho m \cdot x+t$ for all $t \in F$ and all $m \in Z_{+}$.

Let $Q(a)$ denote the $\rho$-class containing $a \in P$. Let $\mathrm{U}_{F}(a)$ or $\mathrm{U}(a)=[Q(a), F]$, i.e., the subsemigroup of $S$ generated by $Q(a)$ and $F$. By (3.1.1) and (3.1.2), we see $Q(a)+F \subseteq Q(a)$. If $s, t \in F$ and $b \in Q(a)$, then $k \cdot b+s=l \cdot b+t$, $\left(k, l \in Z_{+}^{0}\right)$, implies $k=l$ and $s=t$. In fact, if $k>l,(k-l) \cdot b+s=t$ by cancellation, but this is impossible since $F$ is a filter and $b \in P$. Hence $k \leqslant l$. Likewise $k \geqslant l$. Therefore, $k=l$, and hence $s=t$ by cancellation. Thus we have

(3.2) Each element of $Q(a)+F$ has a unique expression as the sum of an element of $Q(a)$ and an element of $F$.

As defined in $\S 1, \bar{X}$ denotes the unitary closure of $X$ and $\tilde{X}$ denotes the filter closure of $X$.

LEMMA 3.3.

$$
\mathrm{U}(a)=\left\{x \in S: m \cdot x \in \overline{\mathrm{P}(a)} \text { for some } m \in Z_{+}\right\}
$$

and

$$
\overline{\mathrm{P}(a)} \subseteq \mathrm{U}(a)=\overline{\mathrm{U}(a)} \subseteq \widetilde{\mathrm{P}(a)}=\widetilde{\mathrm{U}(a)}
$$

Proof. (3.3.1) If $x \in F$, then $x \in \mathrm{P}(a) \subseteq \overline{\mathrm{P}(a)}$. If $x \in Q(a)$, then $m \cdot x$ $+s=n \cdot a+t$ for some $s, t \in F$, some $m, n \in Z_{+} ;$hence $m \cdot x \in \overline{\mathrm{P}(a)}$. Therefore $U(a)$ is contained in the set at the right-hand side. To prove the other direction, let $m \cdot x \in \overline{\mathrm{P}(a)}$. By definition, $n \cdot a+s+m \cdot x=l \cdot a+t$ for some $s, t \in F$, and some $n, l \in Z_{+}^{0}$. Suppose $n>l$. Then $a+z=t$ for some $z \in S$. This contradicts $a \in P$. Hence $n \leqslant l$. If $n=l$, then $x \in F$. If $n<l$, then $m$ • $x+s=(l-n) \cdot a+t$ which implies $x \in Q(a)$, hence $x \in \mathrm{U}(a)$. Thus we have (3.3.1).

(3.3.2) It immediately follows from (3.3.1) and the definition that $\overline{\mathbf{P}(a)}$ $\subseteq \mathrm{U}(a) \subseteq \widetilde{\mathrm{P}(a)}$. Taking their filter closures, we get $\tilde{\mathrm{P}(a)}=\tilde{\mathrm{U}(a)}$ by (1.2.3). It remains to show $\overline{\mathrm{U}(a)} \subseteq \mathrm{U}(a)$. Let $x \in \overline{\mathrm{U}(a)}$. Then $b=c+x$ for some $b, c \in$ $\mathrm{U}(a)$. By (3.3.1), we can choose $m \in Z_{+}$such that $m \cdot b, m \cdot c \in \overline{\mathrm{P}(a)}$. Since $m \cdot b=m \cdot c+m \cdot x$ and $\overline{\mathrm{P}(a)}$ is unitary by (1.2.2), we see that $m \cdot x \in \overline{\mathrm{P}(a)}$. So $x \in \mathrm{U}(a)$. Therefore $\overline{\mathrm{U}(a)} \subseteq \mathrm{U}(a)$. This completes the proof. $\square$

Let $T$ be a subsemigroup of a commutative cancellative semigroup $S$ and let $h \in \operatorname{Hom}\left(T, \mathbf{R}_{+}^{0}\right)$. We say that $\langle T, h\rangle$ satisfies the $B$-condition (boundedness condition) in $S$ if, for each $a \in S$, there is an $M \in R_{+}^{0}$ such that

(B) $x, y \in T, m \in Z_{+}^{0}$ and $y+m \cdot a \in x+S$ implies $h(x)-h(y) \leqslant m \cdot M$. 
Here $M$ is required to be independent of $x, y$ and $m$. The notation $0 \cdot a+y$ expresses $y$ itself, and hence the $B$-condition implies the $K$-condition. The $B$ condition is equivalent to the combination of the $K$-condition and the following:

For each $a \in S$, the set

$$
\left\{m^{-1}[h(x)-h(y)]: x, y \in T, m \in Z_{+}, y+m \cdot a \in x+S\right\}
$$

is bounded.

LEMMA 3.4. The following are equivalent:

(3.4.1) $\langle T, h\rangle$ satisfies the K-condition in $S$.

(3.4.2) $h$ is extended to $\bar{h} \in \operatorname{Hom}\left(\bar{T}, \mathbf{R}_{+}^{0}\right)$.

(3.4.3) $h$ is extended to $\tilde{h} \in \operatorname{Hom}\left(\widetilde{T}, \mathbf{R}_{+}^{0}\right)$.

Proof. (3.4.1) $\Rightarrow(3.4 .2)$. This follows from Theorem 2.1 since $T$ is cofinal in $\bar{T}$.

(3.4.2) $\Rightarrow$ (3.4.3). Since $\bar{T}$ is unitary cofinal in $\widetilde{T}$ by (1.2.4), $\bar{h}$ can be extended to $\widetilde{h} \in \operatorname{Hom}\left(\widetilde{T}, \mathbf{R}_{+}^{0}\right)$ by Corollary 2.6 , and hence $h$ is extended to $\widetilde{T}$.

(3.4.3) $\Rightarrow$ (3.4.1). This is obvious from the definition of $\widetilde{T}$. $\square$

LemMa 3.5. Let $T$ be a filter of $S, T \neq S$, and let $h \in \operatorname{Hom}\left(T, \mathbf{R}_{+}^{0}\right)$. Let a $\in S \backslash T$ and $r \in \mathbf{R}_{+}^{0}$. Define $h_{r}: \mathbf{P}_{T}(a) \rightarrow \mathbf{R}_{+}^{0}$ by

$$
h_{r}(x)= \begin{cases}m \cdot r+h(s) & \text { if } x=m \cdot a+s \text { where } m \in Z_{+}^{0}, s \in T, \\ m \cdot r & \text { if } x=m \cdot a \text { where } m \in Z_{+} .\end{cases}
$$

Every extension of $h$ to $\mathbf{P}_{T}(a)$ is obtained as $h_{r}$ for some $r \in \mathbf{R}_{+}^{0}$.

Proof. Since the expression of $x$ is unique, $h_{r}$ is well defined. The proof of the lemma is easy. $\square$

THEOREM 3.6. Let $T$ be a filter of a commutative cancellative semigroup $S, T \neq S$, and let $h \in \operatorname{Hom}\left(T, \mathbf{R}_{+}^{0}\right)$. Then the following are equivalent:

(3.6.1) $\langle T, h\rangle$ satisfies the B-condition.

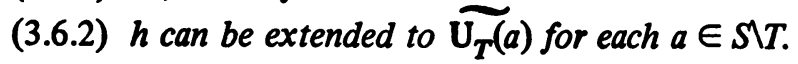

(3.6.3) For each $a \in S \backslash T,\left\langle\mathbb{P}_{T}(a), h_{r}\right\rangle$ satisfies the $K$-condition in $S$ for some $r \in \mathbf{R}_{+}$.

Proof. (3.6.1) $\Rightarrow$ (3.6.2). Choose $r \in \mathbf{R}_{+}$such that

$$
r \geqslant \operatorname{Sup}\left\{m^{-1}[h(x)-h(y)]: y+m \cdot a \in x+S\right\}
$$

and then define $\overline{\bar{h}}: \mathbf{U}_{T}(a) \rightarrow \mathbf{R}_{+}^{0}$ by 


$$
\bar{h}(b)= \begin{cases}h(b) & \text { if } b \in T, \\ \frac{m \cdot r+h(s)-h(t)}{n} & \text { if } b \in Q(a) \text { and } n \cdot b+t=m \cdot a+s \\ & \text { for some } s, t \in T .\end{cases}
$$

By the choice of $r, \overline{\bar{h}}(b) \geqslant 0$ for all $b \in \mathrm{U}_{T}(a)$. To show $\overline{\bar{h}}$ is well defined, let $n \cdot b+t=m \cdot a+s$ and $n_{1} \cdot b+t_{1}=m_{1} \cdot a+s_{1}$ where $s, t, s_{1}, t_{1} \in T$. Then we have $\left(m n_{1}\right) \cdot a+n_{1} \cdot s+n \cdot t_{1}=\left(m_{1} n\right) \cdot a+n \cdot s_{1}+n_{1} \cdot t$ which implies $m n_{1}=m_{1} n$ and $n_{1} \cdot s+n \cdot t_{1}=n \cdot s_{1}+n_{1} \cdot t$ since $T$ is a filter. Then it follows that $\bar{h}$ is well defined. Next we show that $\overline{\bar{h}}$ is a homomorphism. If $b, c \in Q(a)$, then $n \cdot b+t=m \cdot a+s, k \cdot c+u=l \cdot a+v$ for some $t, s$, $u, v \in T, n, m, k, l \in Z_{+} ;$so $(n k) \cdot(b+c)+k \cdot t+n \cdot u=(m k+l n) \cdot a+k$ $\cdot s+n \cdot v$ which implies $\overline{\bar{h}}(b)+\overline{\bar{h}}(c)=\overline{\bar{h}}(b+c)$. If $b \in Q(a)$ and $c \in T$, then $n \cdot b+t=m \cdot a+s$ and $n \cdot(b+c)+t=m \cdot a+s+n \cdot c$, so the same result follows, and we see $\overline{\bar{h}}(b)+\overline{\bar{h}}(c)=\overline{\bar{h}}(b+c)$ for all $b, c \in \mathbf{U}_{T}(a)$. Since $\mathrm{U}_{T}(a)$ is unitary cofinal in $\widetilde{\mathrm{U}_{T}(a)}$ by Lemma 3.3 and (1.2.4), $\bar{h}$ can be extended to $\tilde{h} \in \operatorname{Hom}\left(\widetilde{\mathbf{U}_{T}}(a), \mathbf{R}_{+}^{0}\right)$ by Corollary 2.6 .

(3.6.2) $\Rightarrow(3.6 .1)$. Let $x, y \in T$ and assume $b+x=m \cdot a+y$ for some $b \in S$. Hence $b \in Q(a)$. By assumption, $h$ is extended to $\tilde{h} \in \operatorname{Hom}\left(\widetilde{\mathbf{U}_{T}(a)}, \mathbf{R}_{+}^{0}\right)$, and $m \cdot \tilde{h}(a)+h(y)-h(x)=\tilde{h}(b) \geqslant 0$ which implies the conclusion.

(3.6.2) $\Rightarrow$ (3.6.3). By Lemma 3.3, $\widetilde{U_{T}(a)}=\widetilde{\mathbf{P}_{T}(a)}$. Let $\widetilde{h}$ be the extension of $h$ to $\widetilde{U_{T}(a)}$. Then $\tilde{h} \mid \mathbb{P}_{T}(a)=h_{r}$ for some $r \in \mathbf{R}_{+}^{0}$ by Lemma 3.5. By Lemma 3.4, $\left\langle P_{T}(a), h_{r}\right\rangle$ satisfies the $K$-condition.

(3.6.3) $\Rightarrow$ (3.6.2). Again use Lemma 3.3 and Lemma 3.4. $\square$

4. Examples. Examples 4.1, 4.2 and 4.3 show that the K-condition does not imply the B-condition; Theorem 2.1 is not true in general if $T_{0}$ is not cofinal.

EXAMPLE 4.1. A commutative cancellative idempotent-free semigroup $S$ is defined by

$$
S=\left\{(x, y): y \in Z_{+} \text {if } x=0, y \in Z \text { if } x \in Z_{+}\right\} .
$$

in which the operation is

$$
(x, y)+(z, u)=(x+z, y+u) \text {. }
$$

Let $T_{0}=\left\{(0, y): y \in Z_{+}\right\} . T_{0}$ is not cofinal in $S$. Define $h_{0} \in \operatorname{Hom}\left(T_{0}, \mathbf{R}_{+}^{0}\right)$ by $h_{0}(0, y)=y$. Suppose $h_{0}$ is extended to $h \in \operatorname{Hom}\left(S, \mathbf{R}_{+}^{0}\right)$. Let $x_{0} \in Z_{+}$be fixed and let $\lambda=h\left(x_{0}, 0\right), \lambda \in \mathbf{R}_{+}^{0}$. Choose $y \in Z_{+}$such that $y>\lambda$. Then $h\left(x_{0},-y\right)+h(0, y)=h\left(x_{0}, 0\right)=\lambda$, hence $h\left(x_{0},-y\right)=\lambda-y \geqslant 0$. This is a contradiction. Therefore $h_{0}$ cannot be extended to any element of $\operatorname{Hom}\left(S, \mathbf{R}_{+}^{0}\right)$. 
Let $(b, c) \in S \backslash T_{0}$ be fixed. Take arbitrarily $x, y, m \in Z_{+}$. Let $p=m b$, and $q=m c+y-x$. Then $p \in Z_{+}, q \in Z$ and $(0, x)+(p, q)=m \cdot(b, c)+$ $(0, y)$. Since $(x-y) / m=\left(h_{0}(0, x)-h_{0}(0, y)\right) / m$ can be arbitrarily large, $\left\langle T_{0}\right.$, $\left.h_{0}\right\rangle$ does not satisfy the $B$-condition. Since $T_{0}$ is a filter of $S$, the $K$-condition is satisfied by $\left\langle T_{0}, h_{0}\right\rangle$.

EXAMPLE 4.2. Let $S=\left\{(x, y): x \in Z_{+}^{0}, y \in Z, y \geqslant 1-x^{2}\right\}$ and define $T_{0}$ and $h_{0}$ by

$$
T_{0}=\left\{(0, y): y \in Z_{+}\right\}, \quad h_{0}(0, y)=y .
$$

$T_{0}$ is not cofinal but is a filter in $S$. Suppose that $h_{0}$ can be extended to a homomorphism $h$ of $S$ into $\mathbf{R}_{+}$. For each $n \in Z_{+}^{0}$, let

$$
\varphi(n)=h_{0}\left(n, 1-n^{2}\right) \text {. }
$$

Then $\varphi(1)+\varphi(n-1)-\varphi(n)=h_{0}(0,2 n-1)=2 n-1$ for each $n \in Z_{+}$. From this recurrence relation, we have

$$
n \varphi(1)+\varphi(0)-\varphi(n)=\sum_{i=1}^{n}(2 i-1)=n(n+1)-n=n^{2} .
$$

Since $\varphi(0)=h(0,1)=1$, it follows that $n \varphi(1)-\varphi(n)=n^{2}-1$. By the assumption $\varphi(n) \geqslant 0$ for all $n \in Z_{+}$, we have

$$
n \varphi(1) \geqslant n^{2}-1 \text { for all } n \in Z_{+}
$$

hence $\varphi(1) \geqslant n-1 / n$ for all $n \in Z_{+}$. This is impossible. It follows that $h_{0}$ cannot be extended to an element of $\operatorname{Hom}\left(S, \mathbf{R}_{+}^{0}\right)$. We show that the B-condition is not satisfied. Let $m \in Z_{+}, m>1$, and choose $y, z \in Z_{+}$such that $z-y=m^{2}$ - 1. Then

$$
(0, z)+\left(m, 1-m^{2}\right)=m \cdot(1,0)+(0, y)
$$

but $(z-y) / m=m-1 / m$ can be taken arbitrarily large.

EXAMPLE 4.3. Let $\pi$ be the transcendental real number and let $a=\pi / 4$. Then $0<\pi / 4<1$, and $a$ is transcendental over the field $R$ of rational numbers. If $a_{k}=a^{k}(k=1,2, \ldots)\left(a^{k}\right.$ is the usual $k$ th power of $\left.a\right)$, then $1, a_{1}, a_{2}, \ldots$ are linearly independent over $R$ and $0<a_{k}<1(k=1,2, \ldots)$. Let $T_{0}$ be the additive semigroup of $R_{+}$generated by $a_{1}, \ldots, a_{k}, \ldots, T_{0}$ is actually a free commutative semigroup over $a_{1}, \ldots, a_{k}, \ldots$. Let $b_{k}=1-a_{k}>0(k=$ $1,2, \ldots)$, and let $S$ be the subsemigroup of $R_{+}$generated by $T$ and $b_{1}, b_{2}, \ldots$, $b_{k} \ldots . T_{0}$ is a filter of $S$. Define $h_{0}: T_{0} \rightarrow R_{+}$by the homomorphism given by $h_{0}\left(a_{k}\right)=k$. Since $T_{0}$ is free, $h_{0}$ is well defined. Then $h_{0}$ cannot be extended to $h \in \operatorname{Hom}\left(S, \mathbf{R}_{+}^{0}\right)$. For suppose $h_{0}$ is extended to $h$. Then $1=a_{k}$ $+b_{k} \in S$, which implies $h(1)=h\left(a_{k}+b_{k}\right) \geqslant h\left(a_{k}\right)=k$ for all $k$. This is a 
contradiction. Finally we show that $\left\langle T_{0}, h_{0}\right\rangle$ does not satisfy the B-condition. Let $m \in Z_{+}$and $a_{k} \in T$. As $a_{k}\left|1, a_{k}\right| m$ and so $a_{k} \mid\left(m+a_{i}\right)$ in $S$ for all $m \in Z_{+}$, all $a_{i}, a_{k} \in T$. Then

$$
\left(h_{0}\left(a_{k}\right)-h_{0}\left(a_{i}\right)\right) / m=(k-i) / m
$$

is not bounded.

ACKNOWLEDGEMENT. The authors thank the referee for his kind suggestion on this paper.

ADDENDUM. The assumption of cancellation does not restrict our discussion by the following reason. Let $S$ be a commutative semigroup, $S_{0}$ the greatest cancellative homomorphic image of $S$, and $g_{0}: S \rightarrow S_{0}$ the homomorphism. If $f$ is a homomorphism of $S_{0}$ into $\mathbf{R}_{+}^{0}$, then $h=f g_{0}$ is a homomorphism of $S$ into $\mathbf{R}_{+}^{0}$. Every homomorphism $h$ of $S$ into $\mathbf{R}_{+}^{0}$ can be obtained in this manner. Accordingly the results in this paper are extended to the case in which cancellation is not assumed.

\section{REFERENCES}

1. A. H. Clifford and G. B. Preston, The algebraic theory of semigroups, vol. II, Math. Surveys, no. 7, Amer. Math. Soc., Providence, R. I., 1967. MR 36 \#1558.

2. Y. Kobayashi, Homomorphisms on $N$-semigroups into $R_{+}$and the structure of $N$. semigroups, J. Math. Tokushima Univ. 7 (1973), 1-20. MR 48 \#3831.

3. M. Petrich, Introduction to semigroups, Merrill, Columbus, Ohio, 1973.

4. M. S. Putcha, Positive quasi-orders on semigroups, Duke Math. J. 40 (1973), 857869. MR 49 \#2998.

5. T. Tamura and H. B. Hamilton, The study of commutative semigroups with greatest group homomorphism, Trans. Amer. Math. Soc. 175 (1972), 401-419. MR 47 \#3581.

DEPARTMENT OF MATHEMATICS, NORTH CAROLINA STATE UNIVERSITY, RALEIGH, NORTH CAROLINA 27607

DEPARTMENT OF MATHEMATICS, UNIVERSITY OF CALIFORNIA, DAVIS, CALIFORNIA 95616 